\title{
The Thermal Properties of Gliding arc Plasma Produced by Laboratory Reverse Vortex Flow System
}

\author{
Farah A. Lazem ${ }^{\text {a*, Hammad R. Humud }}{ }^{\mathrm{b}}$ \\ Department of Physics, College of Science, University of Baghdad, Baghdad, Iraq \\ ${ }^{b}$ E-mail: dr.hammad6000@yahoo.com \\ a*Corresponding author: farah.lazem1204@sc.uobaghdad.edu.iq
}

\begin{abstract}
A low-cost reverse flow plasma system powered by argon gas pumping was built using homemade materials in this paper. The length of the resulting arc change was directly proportional to the flow rate, while using the thermal camera to examine the thermal intensity distribution and demonstrating that it is concentrated in the centre, away from the walls at various flow rates, the resulting arc's spectra were also measured. The results show that as the gas flow rate increased, so did the ambient temperature. The results show that the medium containing the arc has a maximum temperature of 34.1 ${ }^{\circ} \mathrm{C}$ at a flow rate of $14 \mathrm{~L} / \mathrm{min}$ and a minimum temperature of $22.6{ }^{\circ} \mathrm{C}$ at a flow rate of $6 \mathrm{~L} / \mathrm{min}$.
\end{abstract}

Article Info.

Keywords:

Non-thermal plasma, gliding arc plasma, reverse vortex flow, thermal camera

\section{Article history:}

Received: May 30, 2021

Accepted: Jul. 03, 2021

Published: Sep. 01,2021

\section{Introduction}

Plasma systems are classified into two types: thermal and non-thermal [1], each with advantages and disadvantages. Thermal plasma systems have disadvantages in that they generate plasma without selectivity agitation, which is dangerous at very high temperatures. They also have cooling requirements and problems with electrode wear bows [2]. These features, in turn, lead to limited energy efficiency and applicability to thermal plasma sources [3]. Operating pressure and energy levels for non-thermal discharges are usually limited, which limits the production rates for industries [4]. Large conventional thermal and non-thermal discharges cannot simultaneously provide a high level of non-thermal discharges balance [5], elevated electron temperature and high electron density. However most chemical applications require high potential power for reactor productivity efficiency and high degree of imbalance to support selective chemical processes [6]. This is the reason why one of the vital challenges of modern discharge physics is a hybrid plasma system that combines the benefits of both thermal and non-thermal plasma systems. By creating high-power, high-pressure discharges that can generate unbalanced plasma [7], used in a variety of exhaust gas cleaning applications [8], Pollution control, fuel diversion, hydrogen gas production, and surface treatments are all examples of surface treatments [9]. In this paper a reactor was built with available and inexpensive materials with a rotating gliding arc plasma and a threedimensional cylinder that works by compressing the gas through side transverse holes with the axis of the circle in order to divert heat from the walls and throw the energy into the core of the reactor.

\section{Experimental work}

\subsection{Mechanical design}

The proposed system is made up of three major components: a base, a Teflon cover, and a Pyrex glass cylinder that serves as a plasma transport medium between the base and the cover. Fig. 1 depicts an outline of the proposed system. 


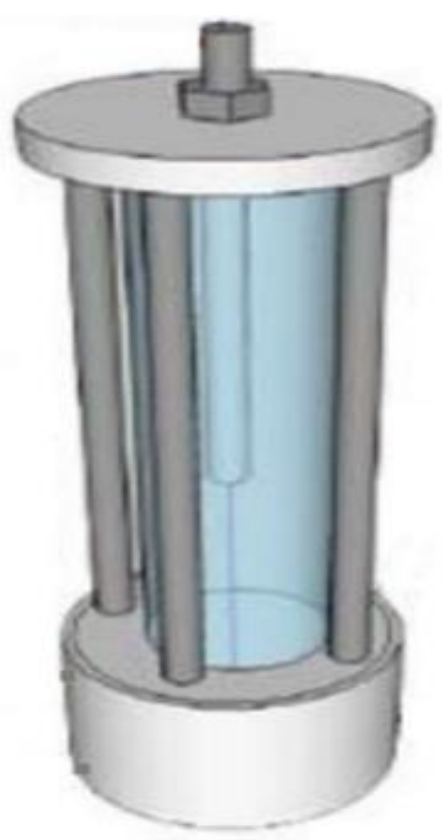

(a)

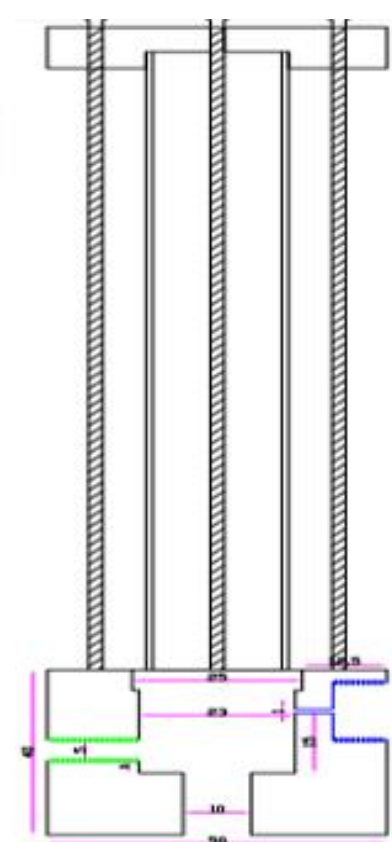

(b)

Figure 1: Reactor design: (a) scheme of the proposed reactor, (b) design Diagram.

The system's total height with its three parts is $190 \mathrm{~mm}$. The hollow base has three different diameters. Four $1 \mathrm{~mm}$ diameter gas pump outlets are tangent to the bore edge and $15 \mathrm{~mm}$ above its base. These enable the formation of the plasma vortex. These outlets are connected to the gas system via nozzles with a diameter of $5 \mathrm{~mm}$. This enables the installation of a copper electrode in the cavity, which is also used for electrical conduction. At the base, a copper wire is connected from the outside to a power source and from the inside to a copper disk, which serves as the first pole. The second pole is a gear-shaped iron pole that can be moved up and down with the help of a DC motor that is controlled from a distance. The third component of the system is the cylinder cover, which measures $10 \mathrm{~mm}$ in length and $50 \mathrm{~mm}$ in diameter. It has a $5 \mathrm{~mm}$ hole in the centre where the wire exits. There are also four iron poles that hold the pieces together.

\subsection{Thermal properties}

The unbalanced plasma is referred to as a non-thermal plasma. In this plasma, the temperature of the electron is 100-1000 times higher than the temperature of the neutral gas [10]. In this way, light and dynamic particles (electrons) create a chemically reactive medium [11]. While the gas is kept at room or slightly elevated temperatures, thermal damage to the environment is avoided. There are different techniques for measuring plasma surface temperature using several measuring instruments [12].

\subsection{Thermal camera}

A thermal camera type S/N 90002261140 was used, as shown in Fig. 2. The principle of operation of the camera is to track the desired body temperature [13]. The thermal imaging camera features a unique lens that allows infrared energy to pass through it and then strike the focused light, and then a sensor that scans the information and extracts from several thousand, the field of view through this process is known as a complex temperature pattern. Where the process of creating a heat plan development of the film takes only few seconds. The thermogramturns into electrical impulses that are directed to the pulse of the signal processing unit, which translates the information into 
files. The generated visual data are displayed as different colours related to the amount of infrared energy emitted from the mixture. Fig. 3 represents a schematic diagram of the camera's internal steps.
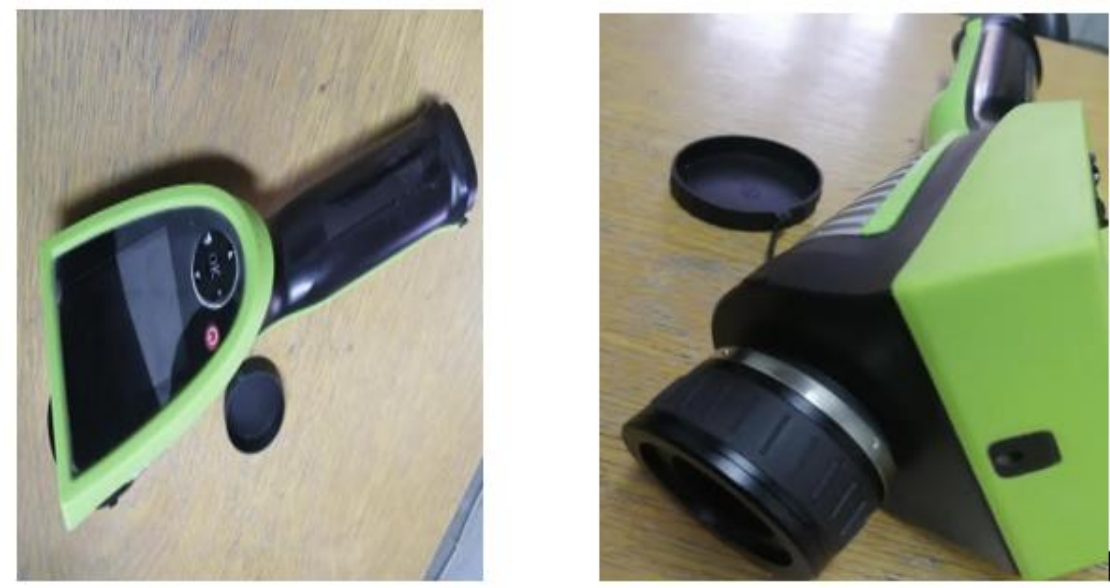

Figure 2: Thermal camera used.

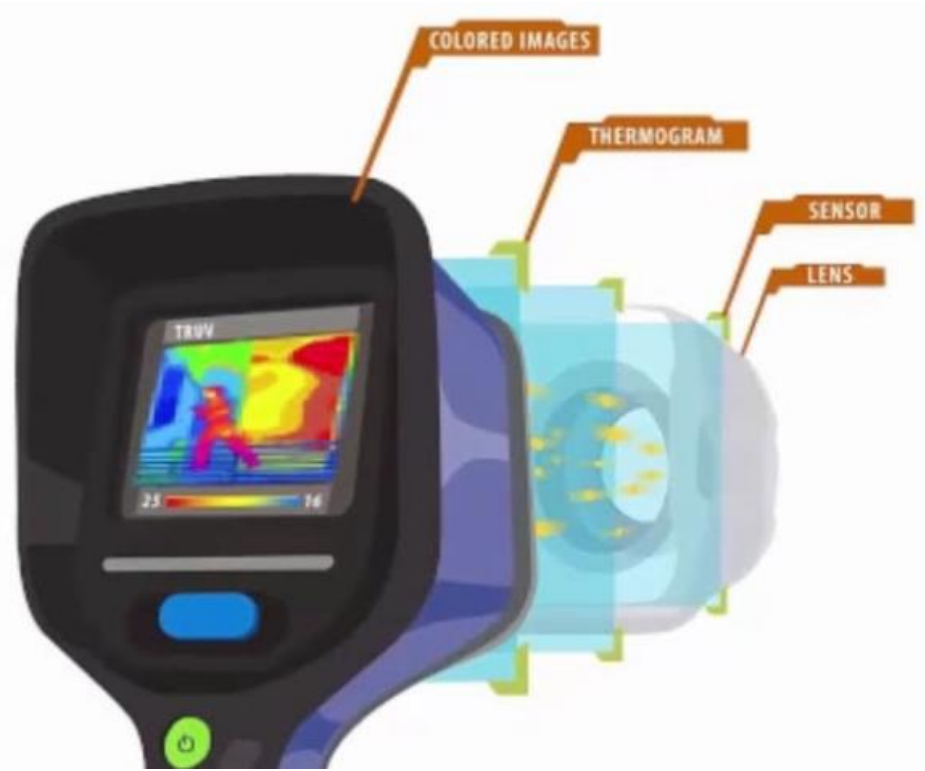

Figure 3: A diagram showing the parts of the thermal camera.

\subsection{Optical emission spectroscopy}

One of the methods for diagnosing plasmas is optical emission spectroscopy [14, 15]. It is used to learn about the nature of plasma, including its chemical composition and types, as well as the gases that comprise the plasma [16]. Fig.4 shows the system for spectrometry.

The signal is collected and entered into the device (1) via a fibre-optic cable, which diffuses a rainbow of colours through pointed diffraction (2). Light scattering is observed on a series of photodiodes (3), each of which only responds to constriction, or the wavelength range that affects it. A dual-charge device (CCD) is connected to the diodes (4). Photodiodes take advantage of the photoelectric effect in the valley formed by the semiconductor and the metal. In the cell, voltage is generated by the electron transition coil. The voltage of the diode is converted into digital form, and the resulting numbers are sent to a dedicated computer that is running a spectrum analysis program. The spectrum is immediately displayed on the screen and saved in the computer files. 
The individual test only takes a fraction of a second, after which the diodes can be emptied to be ready for the second scan.

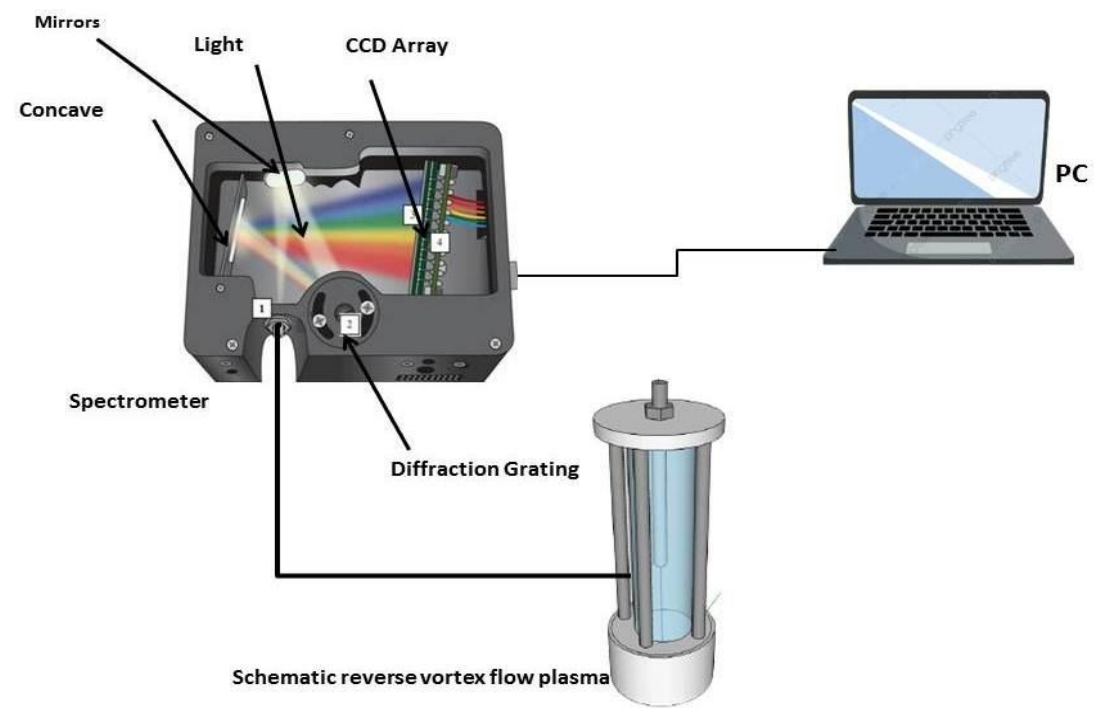

Figure 4: Scheme showing the spectrometer used.

\section{Results and discussion}

A vortex forms inside the reactor as a result of the high-speed gas being pumped through the transverse nozzles (to ensure the gas is transferred from one hole to the other). The reactor must be cylindrical (that is, have a diameter). Initially, argon gas was pumped into the reactor to ensure reactor operation and to achieve an ideal arc at various flow rates $(6,8,10,12$, and 14) $\mathrm{L} / \mathrm{min}$. Fig.5 depicts the ideal arc that appeared when pure argon gas was pumped.

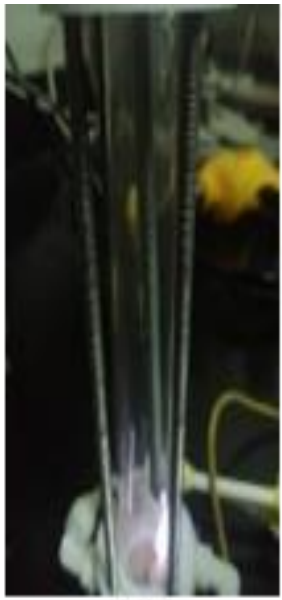

(a)

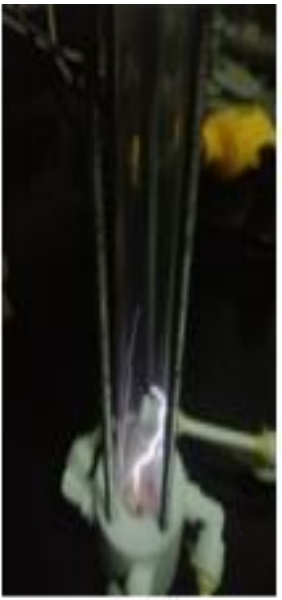

(b)

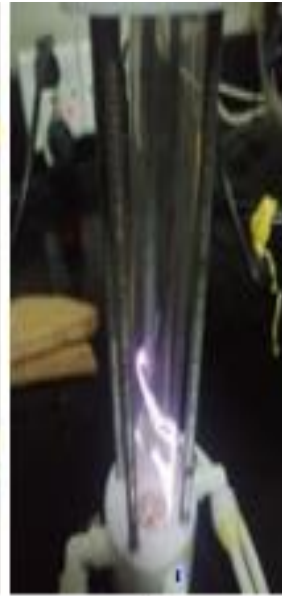

(c)

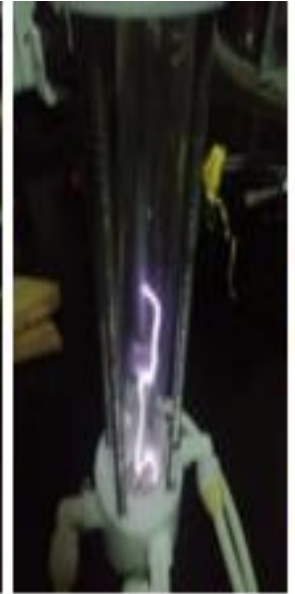

(d)

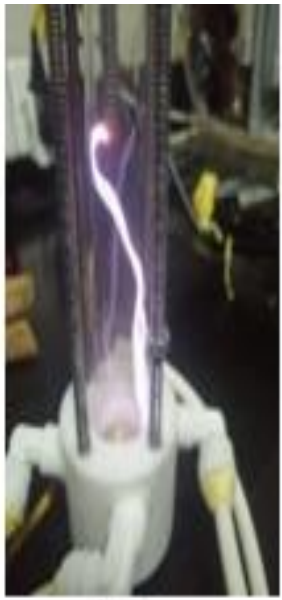

(e)

Figure 5: The maximum plasma length versus different pure argon gas flow rate at: (a) 6 L/min, (b) $8 \mathrm{~L} / \mathrm{min}$, (c) $10 \mathrm{~L} / \mathrm{min}$, (d) $12 \mathrm{~L} / \mathrm{min}$, and (e) $14 \mathrm{~L} / \mathrm{min}$.

The results in Fig.5 show that as the gas flow rate increases, the length and size of the resulting gliding arc change directly. When the gas flow rate was high enough, the resulting eddy currents inside the reactor were pushed to the top, and then collapse occurred, Collapse occur occurred when both the flow rate and the supplied voltage high. 


\subsection{Description of the thermal characterization}

Imaging with a thermal camera reveals the system's thermal nature, and the thermal distribution of the plasma resulted plasma using pure argon gas infusion as shown in Fig.6. The thermal images captured show the intensity of the heat distribution, and see the intensity of the heat confined in the centre can be seen. This demonstrates the significance of the rotating arc in confining heat in the centre and keeping it away from the walls. The temperature of the outer Pyrex surface is indicated by the numbers at the top in thermal image. Table 1 depicts the temperature change as argon gas flow rates vary.

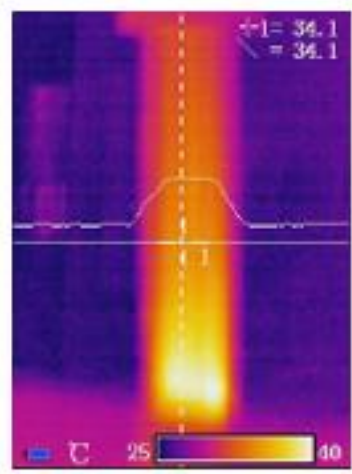

(a)

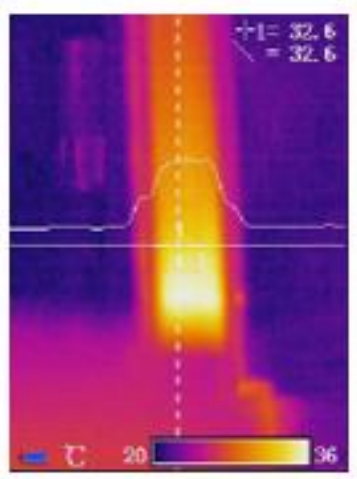

(b)

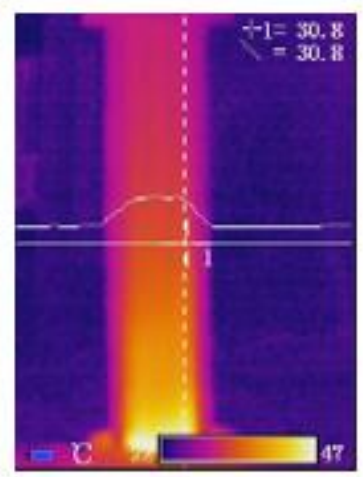

(c)

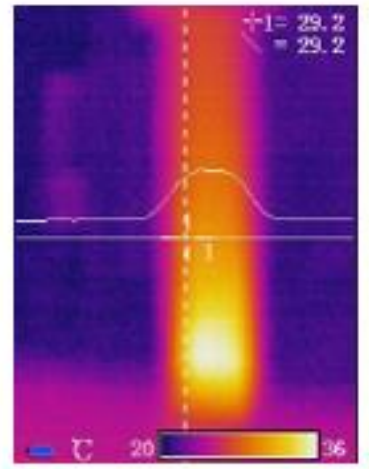

(e)

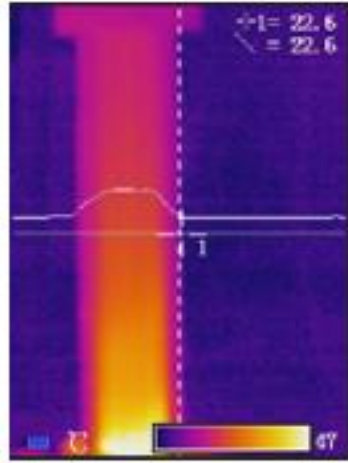

(f)

Figure 6: Thermal camera images for argon gas flow rates of: (a) $14 \mathrm{~L} / \mathrm{min}$, (b) $12 \mathrm{~L} / \mathrm{min}$, (c) $10 \mathrm{~L} / \mathrm{min}$, (d) $8 \mathrm{~L} / \mathrm{min}$, and (e) $6 \mathrm{~L} / \mathrm{min}$.

Table 1: The container medium plasma temperature using various argon gas flows.

\begin{tabular}{c|c}
\hline $\begin{array}{c}\text { Flow rate } \\
(\mathbf{L} / \text { min. })\end{array}$ & $\begin{array}{c}\text { Temperature } \\
\left({ }^{\circ} \mathbf{C}\right)\end{array}$ \\
\hline 6 & 22.6 \\
8 & 29.2 \\
10 & 30.8 \\
12 & 32.6 \\
14 & 34.1 \\
\hline
\end{tabular}

The table above shows that as the gas flow rate increases, so does the temperature of the Pyrex containing the plasma, this is due to an increase in kinetic energy as pumping speed increases. 


\subsection{Optical emission spectroscopy results}

Spectra for argon gas alone were collected. The plasma spectrum produced by argon gas flowing in various flows is depicted in Fig.7 for different gas flow. It can be seen that the intensity of the spectrum increases with increasing gas flow rate, confirming the thermal camera results as the medium containing the arc increases. The goal of the spectroscopic characterization is to show that the intensity of a spectrum increases as the flow rate changes.

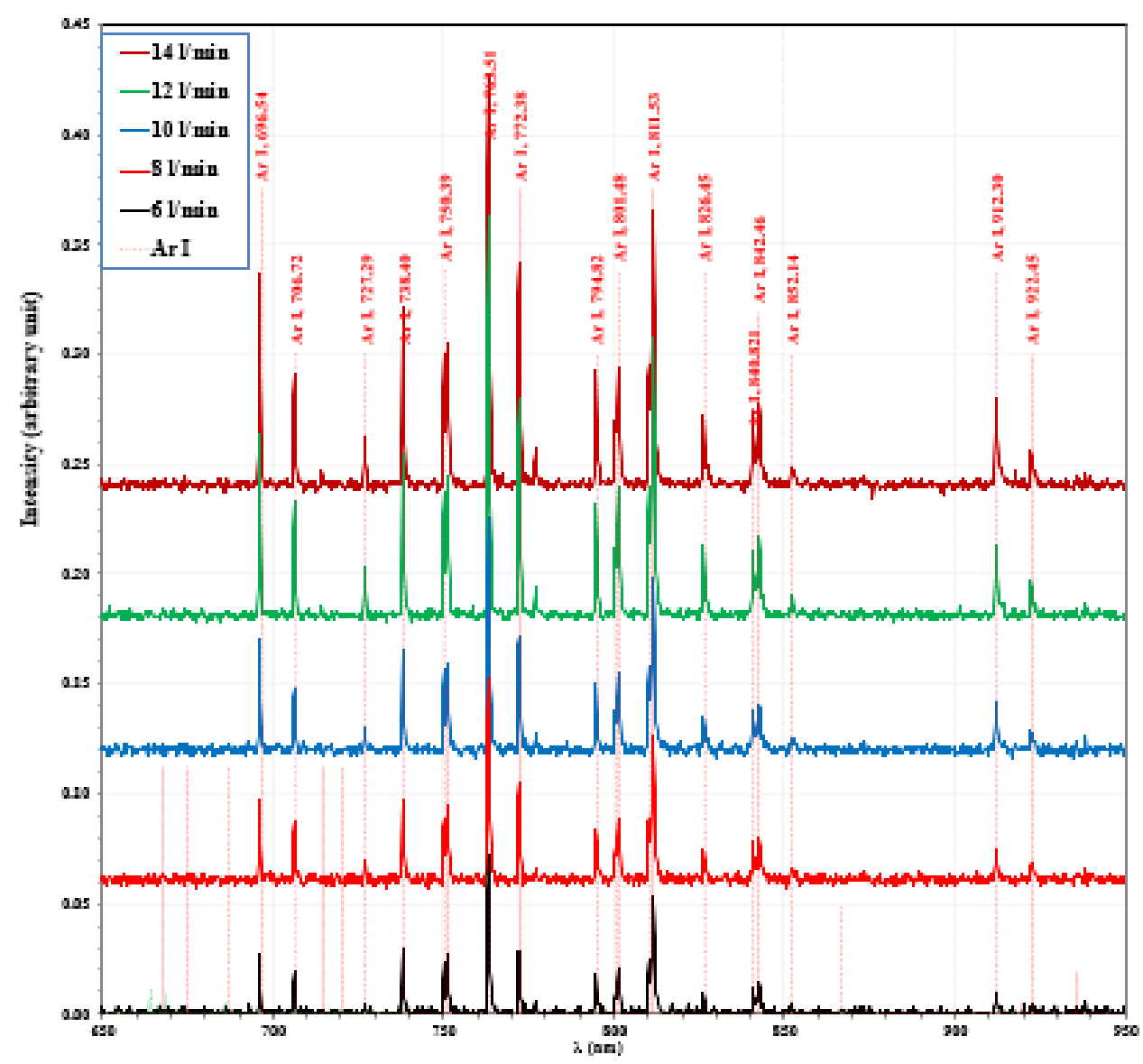

Figure7: Pure argon gas produces a plasma spectrum with varying flow rates.

\section{Conclusions}

In this paper, a system was designed and developed to generate a gliding arc plasma with a rotating reverse flow (RVF-GA) using simple, low-cost materials available in the local market. Argon gas was pumped through the tangent openings and the change of the arc length with the rate of flow was observed. Through the thermal camera, the thermal intensity distribution in the core of the reactor were studied. The temperature of the walls of the cylinder containing the arc was studied, and the success of the tornado arc was proven by confining the temperature only in the middle away from the walls, where it could be touched by the hands. The resulting arc spectrum with various flow rates showed an increase in intensity with each increase in flow rate. The results obtained are promising for uses in numerous industrial and agricultural applications.

\section{Acknowledgements}

The authors would like to thanks University of Baghdad, College of Science, Department of Physics, Dr. Saddam F. Haddawi for technical assistance during the research work. 


\section{Conflict of interest}

Authors declare that they have no conflict of interest.

\section{References}

1. Petitpas G., Rollier J.-D., Darmon A., Gonzalez-Aguilar J., Metkemeijer R., and Fulcheri L., A comparative study of non-thermal plasma assisted reforming technologies. International Journal of Hydrogen Energy, 2007. 32(14): pp. 28482867.

2. Leonelli C. and Mason T.J., Microwave and ultrasonic processing: now a realistic option for industry. Chemical Engineering and Processing: Process Intensification, 2010. 49(9): pp. 885-900.

3. Surowsky B., Schlüter O., and Knorr D., Interactions of non-thermal atmospheric pressure plasma with solid and liquid food systems: a review. Food Engineering Reviews, 2015. 7(2): pp. 82-108.

4. SriBala G., Michiels D., Leys C., Van Geem K.M., Marin G.B., and Nikiforov A., Methane reforming to valuable products by an atmospheric pressure direct current discharge. Journal of cleaner production, 2019. 209: pp. 655-664.

5. Li S., Dang X., Yu X., Abbas G., Zhang Q., and Cao L., The application of dielectric barrier discharge non-thermal plasma in VOCs abatement: A review. Chemical Engineering Journal, 2020. 388: pp. 124275.

6. Chen H.L., Lee H.M., Chen S.H., Chang M.B., Yu S.J., and Li S.N., Removal of volatile organic compounds by single-stage and two-stage plasma catalysis systems: a review of the performance enhancement mechanisms, current status, and suitable applications. Environmental science \& technology, 2009. 43(7): pp. 22162227.

7. Chen J. and Davidson J.H., Model of the negative DC corona plasma: Comparison to the positive DC corona plasma. Plasma chemistry and plasma processing, 2003. 23(1): pp. 83-102.

8. Jaworek A., Krupa A., and Czech T., Modern electrostatic devices and methods for exhaust gas cleaning: A brief review. Journal of electrostatics, 2007. 65(3): pp. 133155.

9. Ricard A., The production of active plasma species for surface treatments. Journal of Physics D: Applied Physics, 1997. 30(16): pp. 2261.

10. Gruntman M., Roelof E.C., Mitchell D.G., Fahr H.J., Funsten H.O., and McComas D.J., Energetic neutral atom imaging of the heliospheric boundary region. Journal of Geophysical Research: Space Physics, 2001. 106(A8): pp. 15767-15781.

11. Kamat P.V., Flumiani M., and Hartland G.V., Picosecond dynamics of silver nanoclusters. Photoejection of electrons and fragmentation. The Journal of Physical Chemistry B, 1998. 102(17): pp. 3123-3128.

12. Iwanaga S., Toberer E.S., LaLonde A., and Snyder G.J., A high temperature apparatus for measurement of the Seebeck coefficient. Review of Scientific Instruments, 2011. 82(6): pp. 063905.

13. Alwazzan M.J., Low cost blood vein detection system based on near-infrared LEDs and image-processing techniques. Polish Journal of Medical Physics and Engineering, 2020. 26(2): pp. 61-67.

14. Bruggeman P., Verreycken T., Gonzalez M.A., Walsh J.L., Kong M.G., Leys C., and Schram D.C., Optical emission spectroscopy as a diagnostic for plasmas in liquids: opportunities and pitfalls. Journal of Physics D: Applied Physics, 2010. 43(12): pp. 124005. 
15. Ahmed A.N., Alwazzan M.J., and Ismael M.A., Study Effects of Pulse Laser Energy on Human Primary Teeth and Extraction Caries Area by Using Image Processing Techniques. NeuroQuantology, 2020. 18(6): pp. 36.

16. Aragón C. and Aguilera J.A., Characterization of laser induced plasmas by optical emission spectroscopy: A review of experiments and methods. Spectrochimica Acta Part B: Atomic Spectroscopy, 2008. 63(9): pp. 893-916.

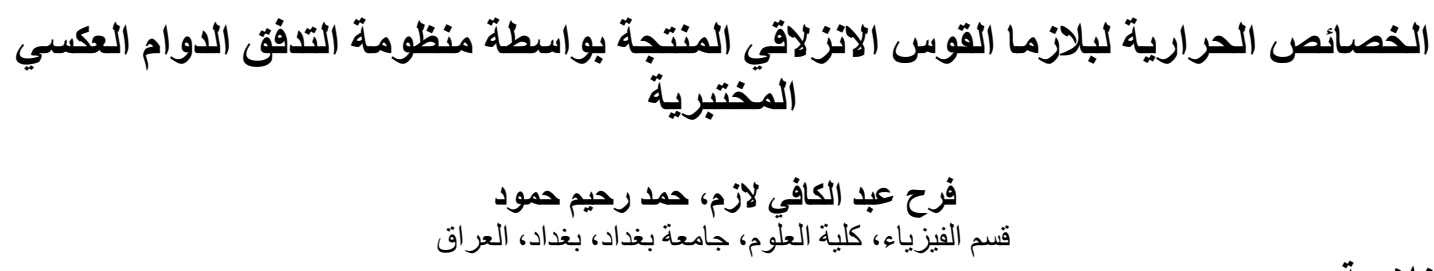

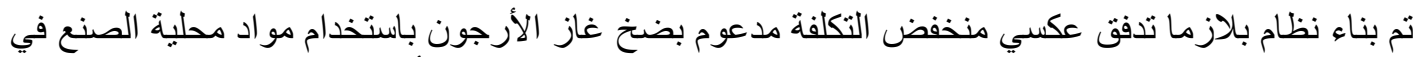

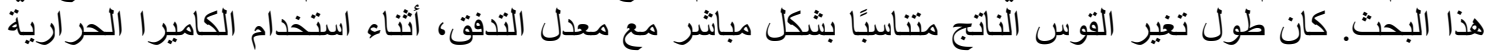

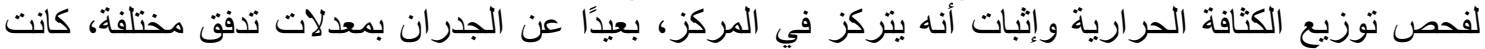

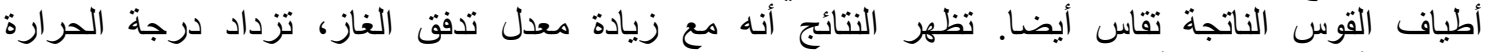

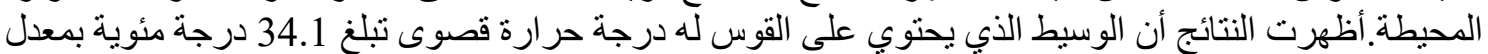

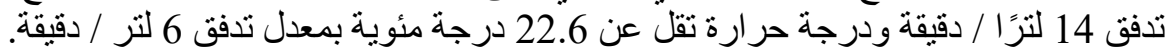

